\title{
Supporting Information: Aligned Stacking of Nanopatterned 2D Materials for High-Resolution 3D Device Fabrication
}

\author{
Jonas Haas ${ }^{1,2}$, Finn Ulrich ${ }^{1,2}$, Christoph Hofer ${ }^{1,2}$, Xiao Wang ${ }^{3}$, Kai Braun ${ }^{4}$, and \\ Jannik C. Meyer ${ }^{1,2}$ \\ ${ }^{1}$ Institute of Applied Physics, Eberhard Karls University of Tuebingen, Auf der Morgenstelle 10, D-72076, Tuebingen, \\ Germany \\ ${ }^{2}$ Natural and Medical Sciences Institute at the University of Tuebingen, Markwiesenstr. 55, D-72770 Reutlingen, Germany \\ ${ }^{3}$ School of Physics and Electronics, Hunan University, Changsha, Hunan 410082, China \\ ${ }^{4}$ Institute of Physical and Theoretical Chemistry, Eberhard Karls University of Tuebingen, Auf der Morgenstelle 18, D-72076, \\ Tuebingen, Germany
}

E-mail: jonas.haas@uni-tuebingen.de, jannik.meyer@uni-tuebingen.de

\section{Preparation of the target substrate}

The preparation of the target substrate is illustrated in Fig. S1. A single post in a FIB lift-out grid is thinned and narrowed by FIB, and a hole is inserted. It is important that this hole is made on the curved surface near the end of the post. 

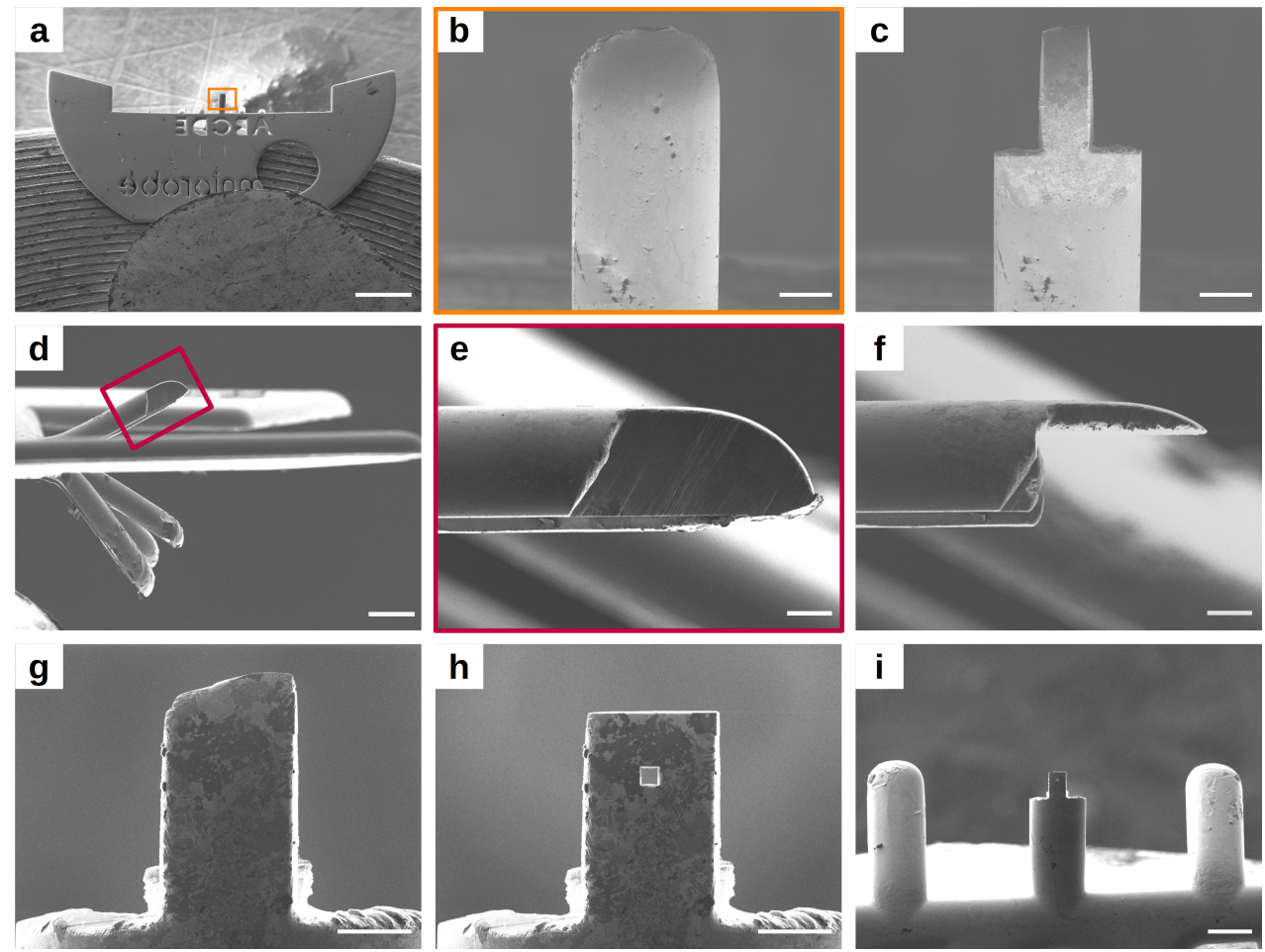

Figure S 1: Preparation of the target substrate. (a) Lift-out grid with one of the five post bent upwards, while the others are bent downwards (not visible). (b) Original shape of the post. (c) Post cut to a smaller width by FIB. (d) Side view of the lift-out grid. Central post (e) before and (f) after milling from the side to reduce the thickness. Top view of the post $(\mathrm{g})$ before and $(\mathrm{h}+\mathrm{i})$ after cutting the central hole for material deposition. Scale bars are (a) $500 \mu \mathrm{m},(\mathrm{b}+\mathrm{c}) 20 \mu \mathrm{m}$, (d+i) $50 \mu \mathrm{m},(\mathrm{e}+\mathrm{f}+\mathrm{g}+\mathrm{h}) 10 \mu \mathrm{m}$. (a+i) are SEM images (SE-detector), (b-h) are FIB images $(30 \mathrm{kV}, 50 \mathrm{pA})$. 


\section{Example of the layer-by-layer assembly}

For the presented nanoscale funnel, ten graphene layers are patterned by EBIE with holes of different sizes and subsequently placed on top of each other in the order of increasing hole size. The stepwise assembly is shown in Fig. S2 as SEM image sequence after each assembled layer.
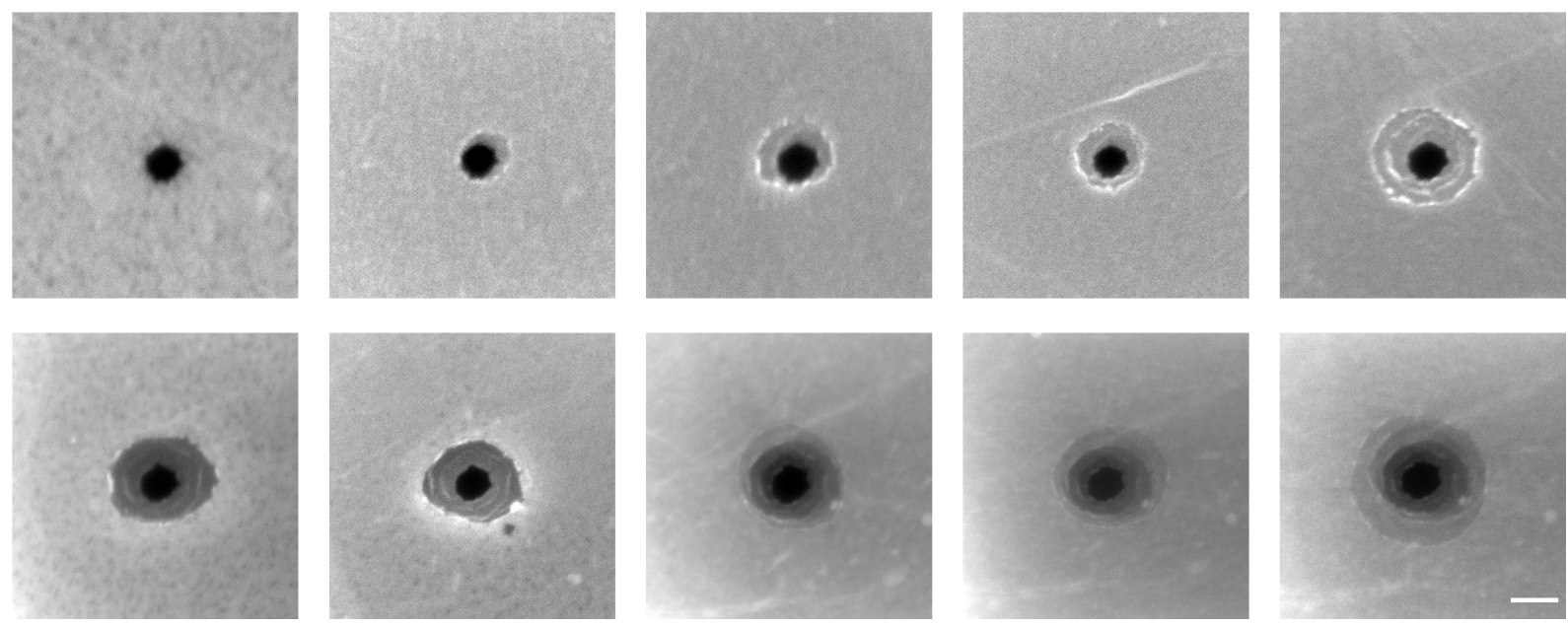

Figure S 2: Layer-by-layer assembly of ten graphene layers with patterned holes of increasing diameter. After placing each layer, a SEM image is taken. The offset, i.e. the distance between the centers of the holes, is determined for each layer with respect to the first layer (smallest hole). Starting from the second layer to the last layer it is $19,18,15,14,16,7,23,35,7 \mathrm{~nm}$ resulting in an average offset for the ten layers of about $17 \mathrm{~nm}$. Scale is $200 \mathrm{~nm}$. 


\section{Patterning non-contiguous structures}

The patterning of a closed ring into graphene by means of EBIE results in an almost freestanding disc, as shown in each first image of Fig. S3a and b. The SEM image sequence shows the effect of additional electron dose. In Fig. S3a, the cut-out structure first folds up and then adheres next to the hole on the graphene membrane, whereas in Fig. S3b the folded structure remains curled at the edge of the hole.
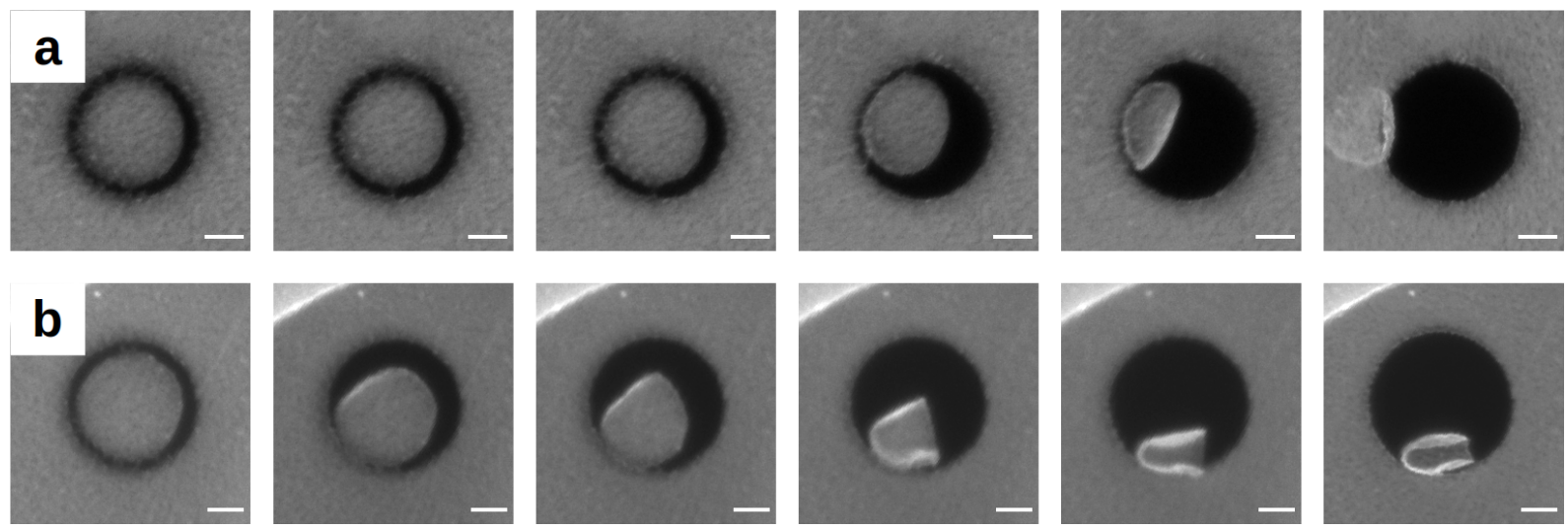

Figure S 3: Patterning "nearly" free-standing discs by EBIE. After an almost isolated structure is formed, additional electron dose causes the structure to (b) adhere next to the hole or (a) to fold up. Scale bars are $250 \mathrm{~nm}$. 


\section{Stability of the patterned nanostructures}

The created nanostructures are investigated for their stability during the stacking process. Fig. S4 shows the transformation of the first hole of the ten layer graphene stack presented in Fig. 5 d-f. This hole was initially patterned by $\mathrm{H}_{2} \mathrm{O}$ assisted EBIE. From the fourth layer onwards, the sample was examined in TEM after each assembly step. The shape of the hole is investigated in each TEM image and the enclosed area is calculated. Example images are shown for the four layer stack (Fig. S4 a) as well as the final ten layer stack (Fig. S4 b). As shown in the colored overlay and in the given table, the size of the smallest hole increases with each assembly step. This is probably due to further etching by the electron beam in SEM during stacking: although the gas-injection system is closed, water is still present in the high-vacuum SEM chamber as well as on the sample surface. Thus, graphene layers are affected by the electron beam not only during patterning but also during stacking.

In addition to a purely organic material like graphene, the heterostructure shown in Fig. 5 a$\mathrm{c}$ is examined for changes while stacking. Fig. S5 shows the patterns $(\mathrm{a}, \mathrm{c})$ before and $(\mathrm{b}, \mathrm{d})$ after

assembling. For the hole patterned in graphene (Fig. S5 a and b), a similar increase of the area is observed as determined for the ten layer stack shown above. The area is attached as text in the respective image. Interestingly, however, the patterns in the TMD layer do not show a change in shape or size. This is determined for the central hole (Fig. S5 c and d) as well as the concentric ring width (see line plot in Fig. S5). Here, the enclosed area as well as the width remain the same when comparing the structures before and after stacking.

This is in agreement with our observation, that TMDs can not be patterned by means of $\mathrm{H}_{2} \mathrm{O}$ assisted EBIE. Fig. 6 shows a $\mathrm{WS}_{2}$ flake (bright triangle) on the amorphous carbon support film of the TEM grids used in this work. With the same parameters used for patterning graphene, it is attempted to pattern the TMD with two lines. However, as can clearly be seen, the TMD is unaffected whereas the much thicker support film already exhibits line-shaped holes.

These results confirms the assumption that the occurred changes in size and shape of the patterned features which only show up in graphene is due to EBIE, assisted by residual water. 

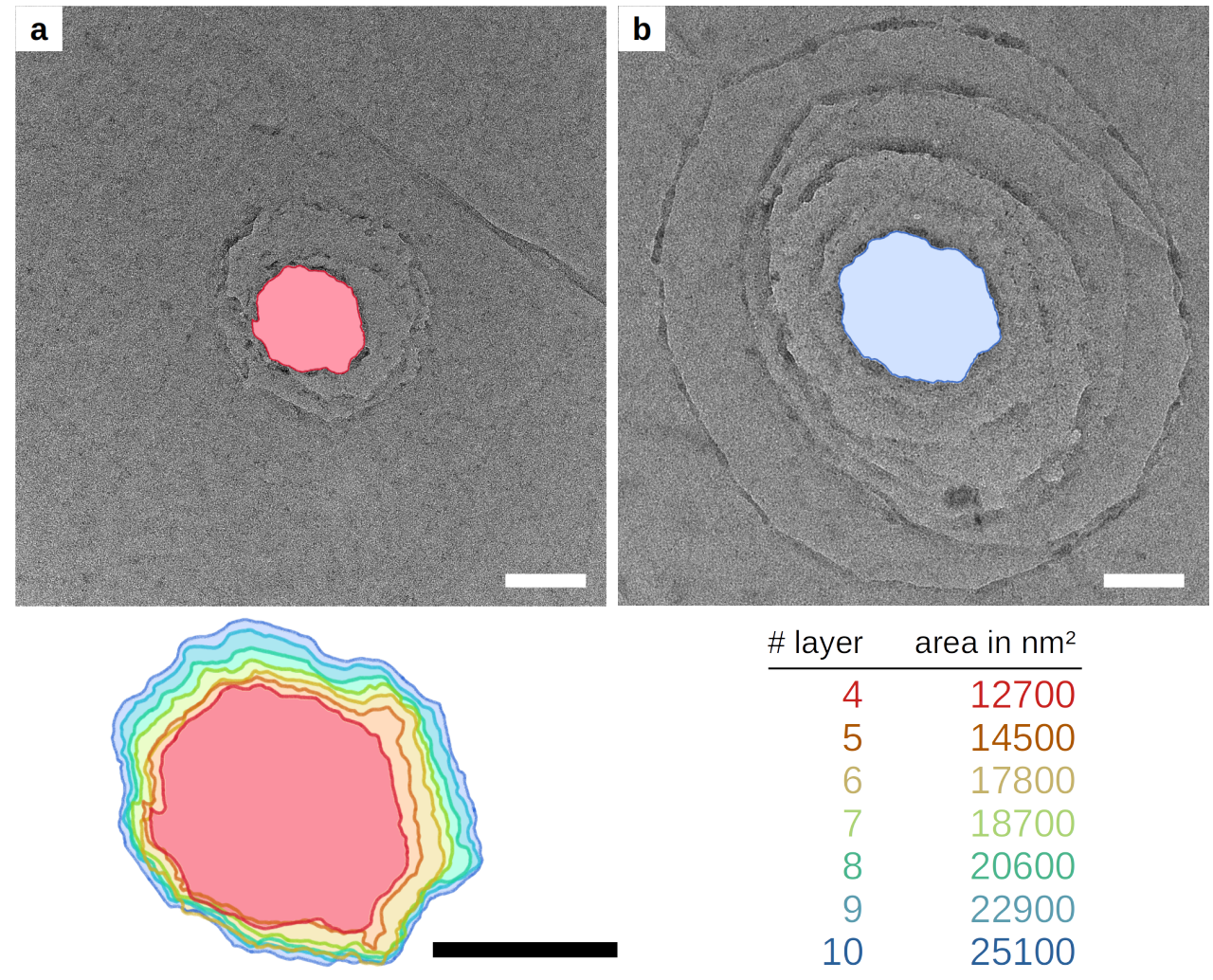

\begin{tabular}{rr} 
\# layer & area in $\mathrm{nm}^{2}$ \\
\hline 4 & 12700 \\
5 & 14500 \\
6 & 17800 \\
7 & 18700 \\
8 & 20600 \\
9 & 22900 \\
10 & 25100
\end{tabular}

Figure S 4: Investigation of the stability of the first hole of the ten layer graphene stack. TEM images after placing (a) four and (b) ten graphene layers, respectively. The colored overlay illustrates the increase of the hole size with each assembly step from red to blue. Scale bars are $100 \mathrm{~nm}$. 

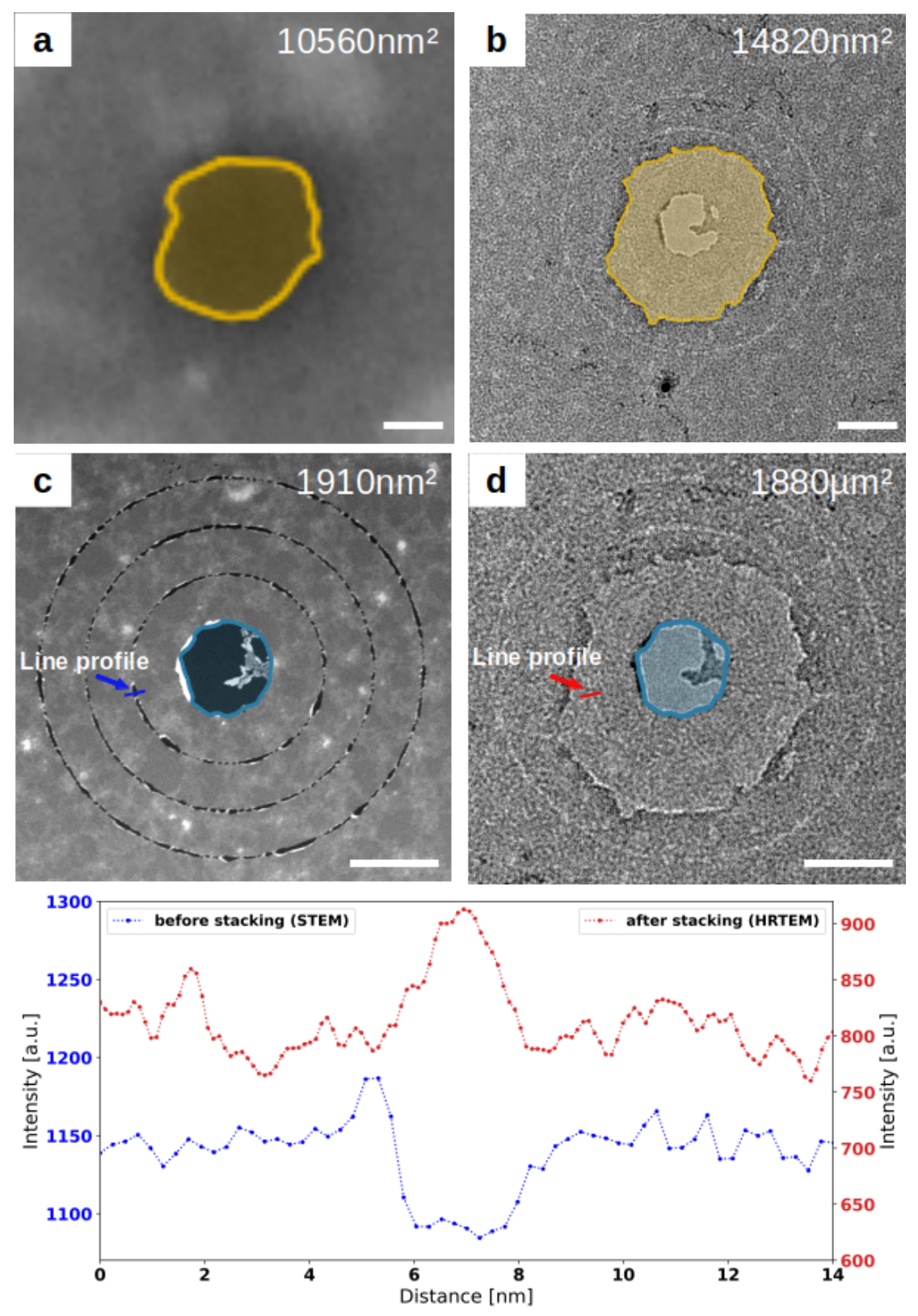

Figure S 5: Comparison of the features in the graphene $+\mathrm{MoS}_{2}$ heterostructure before and after stacking. For better illustration, the compared patterns are colored. The hole in graphene is shown in the (a) SEM image after patterning and the (b) HRTEM image after stacking (here, the slight ellipticity as in Fig. 5 of the main document was compensated by postprocessing). In the latter an increase of the hole size is observed (enclosed area of the hole is given as inset). For the patterns in $\mathrm{MoS}_{2}$ we compared the hole size as well as the line width of the concentric rings. The hole size remains the same when comparing (c) the STEM image after patterning with (d) the HRTEM image after stacking. Also a line plot across the concentric ring shows no change within the scope of the measurement accuracy for the pattern before (blue) and after stacking (red). The contrast change is due to the different imaging method. Scale bars are $50 \mathrm{~nm}$. 


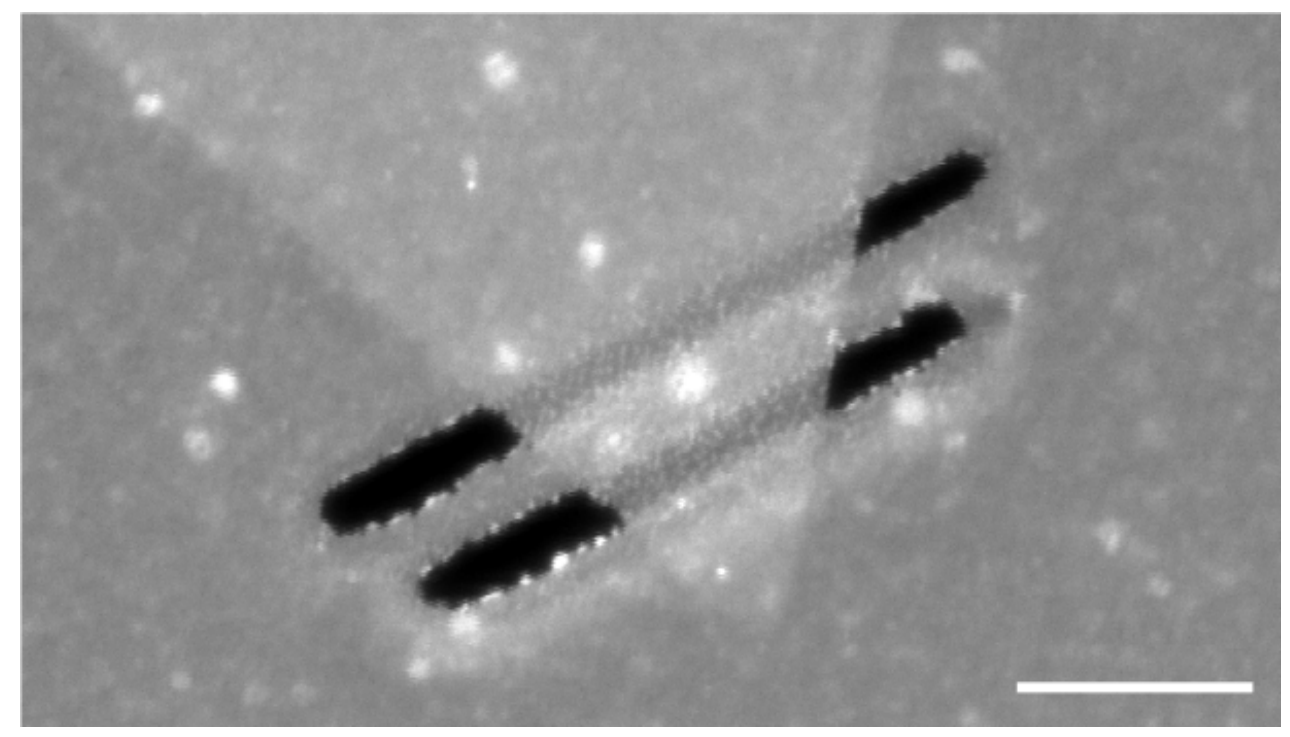

Figure S 6: Patterning $\mathrm{WS}_{2}$ on carbon support film by $\mathrm{H}_{2} \mathrm{O}$ assisted EBIE in SEM. While the $10-12 \mathrm{~nm}$ thick support film is etched through, the TMD flake remains unaffected. Scale bar is $500 \mathrm{~nm}$. 